\title{
Preparation and in vitro Evaluation of Inclusion Complexes of Nelfinavir with Chemically Modified B-cyclodextrins
}

\author{
Shivanand Hiremath ${ }^{1}$ and Ganesh Godge ${ }^{2}$ \\ ${ }^{1}$ Department of Pharmaceutics, PRES'S College of Pharmacy, Sinnar, Nashik- 422101, Maharashtra, India \\ ${ }^{2}$ Department of Pharmaceutics, P.D.V.V.P.F'S College of Pharmacy, Vilad Ghat, Ahmednagar-414111, \\ Maharashtra, India
}

\begin{abstract}
Nelfinavir is a poorly water-soluble antiretroviral drug with relatively low bioavailability. In the present study, the practically insoluble drug, nelfinavir (NFV) and its inclusion complexes with hydroxypropyl- $\beta$ cyclodextrin (HP- $\beta$-CD) were investigated to improve the aqueous solubility and the dissolution rate of the drug, thus enhancing its bioavailability. The phase solubility diagram with HP- $\beta-C D$ was classified as $A_{L}$-type at all temperatures investigated, indicating the formation of higher order complexes. The apparent complexation constants $\left(\mathrm{K}_{1: 1}\right)$ calculated from phase solubility diagram were $145.49,188.45$ and $255.54 \mathrm{M}^{-1}$ at 25,37 and $45 \pm 0.5^{\circ} \mathrm{C}$, respectively. Aqueous solubility and dissolution studies indicated that the dissolution rates were remarkably increased; this could be mainly attributed to the improved solubility and dissolution associated with inclusion complex between drug and HP- $\beta$-CD. Absence of endothermic and characteristic diffraction peaks corresponding to NFV was observed for the inclusion complex in DSC and PXRD. FT-IR study indicated that the presence of intermolecular hydrogen bonds between NFV and HP- $\beta-C D$ in inclusion complex, resulting in the formation of amorphous form.
\end{abstract}

Key words: Nelfinavir, Hydroxypropyl- $\beta$-cyclodextrin, inclusion complex, kneading method, freeze drying.

\section{INTRODUCTION}

Nelfinavir Mesylate (NFV) is used in the treatment of HIV infection in conjunction with other antiretrovirals. ${ }^{1}$ Nelfinavir inhibits the HIV viral protease enzyme which prevents cleavage of the gagpol polyprotein, resulting in noninfectious, immature viral particles. Protease inhibitors (PIs) block the part of HIV called protease. HIV-1 protease is an enzyme required for the proteolytic cleavage of the viral polyprotein precursors into the individual functional proteins found in infectious HIV-1. Nelfinavir binds to the protease active site and inhibits the activity of the enzyme. This inhibition prevents cleavage of the viral polyproteins resulting in the formation of immature non-infectious viral particles. It is practically insoluble in water but well absorbed from oral route. However, due to this disadvantage of poor aqueous solubility this may cause formulation problems and lead to highly variable blood levels,

Correspondence to: Godge Ganesh Raosaheb

E mail: grgodge@yahoo.com

Phone: +919028757508; Fax No. 0241-2422779

Dhaka Univ. J. Pharm. Sci. 11(2): 107-116, 2012 (December) and irreproducible clinical response (therapeutic failure or exaggerated side effects). Therefore, it is important to introduce effective methods to enhance the solubility and dissolution rate of the drug aiming to improve its bioavailability, increase the predictability of the response and/or reduce the dose.

Among the several approaches, complexation with CDs has been widely used to enhance the bioavailability of poorly soluble drugs by increasing the drug solubility, dissolution and/or permeability. ${ }^{2-5}$ Natural CDs are widely explored by many researchers so far, but have limited pharmaceutical applications owing to its limited aqueous solubility $(1.85 \mathrm{~g} / 100 \mathrm{ml}){ }^{6}{ }^{6}$ To overcome this problem, chemically modified $\beta$-CDs have been synthesized examples viz. include heptakis-(2,6-O-dimethyl)- $\beta$ CD (DM- $\beta-C D)$ and hydroxypropyl- $\beta-\mathrm{CD}$ (HP- $\beta$ $\mathrm{CD})$.

Cyclodextrins are cyclic $(\alpha-1,4)$ - linked oligosaccharides of $\alpha$-D glucopyranose, containing a relatively hydrophobic central cavity and hydrophilic outer surface. Due to lack of free rotation about the bonds connecting the glucopyranose units, the 
cyclodextrins are not perfectly cylindrical molecules but are toroidal or cone shaped. Depending upon those models, the primary hydroxyl groups are located on the narrow side of the cone shape, while secondary hydroxyl groups are located on the wider edge. From last two decades, $\mathrm{CDs}$ and their derivatives have been of considerable interest in the pharmaceutical field because of their ability to form complexes with a variety of drug molecules. ${ }^{7}$

Vast literature survey revealed lacks of any data regarding impact of CDs on the solubility profile and dissolution rate of nelfinavir. Thus, the aim of this work was to study the interaction of NFV with the water soluble derivatives of $\beta-C D$ like HP- $\beta-C D$ prepared using various methods, in both solution and solid state, so as to develop a soluble form of the drug as a primary step in the development of nelfinavir tablet formulation. Phase solubility technique and UV spectral shift method were used to investigate the interaction of nelfinavir with cyclodextrins in the solution state.

Solid nelfinavir-chemically modified cyclodextrin binary systems were prepared using different preparation methods. FTIR, XRD and DSC were used to evaluate the physicochemical properties of the prepared systems in order to clarify any interaction between the drug and the used carriers. Invitro dissolution studies of all the prepared systems were carried out to investigate the effect of the molar ratio, $\mathrm{CD}$ type, preparation method on nelfinavir dissolution and the influence of the method of complexation on the physico-chemical properties of the drug-CD complex in order to select the most effective system for improving nelfinavir dissolution properties and in vivo performance.

\section{MATERIALS AND METHODS}

Nelfinavir (Auribindo Pharmaceuticals Ltd., India) was a gift sample from Emcure Pharmaceuticals company (India). $\beta-\mathrm{CD}(\mathrm{MW}=1135 \mathrm{Da})$ and HP- $\beta-C D$ (average degree of substitution; 0.8 and $\mathrm{MW}=1460 \mathrm{Da}$ ) were purchased from SA Pharma Chem. Mumbai. All other chemicals and solvents were of analytical grade and used without further purification.

UV spectroscopic measurements. Spectral shift method was used to illustrate complex formation between nelfinavir and chemically modified CDs in distilled water. ${ }^{8}$ The concentration of NFV was kept constant at $0.04 \mathrm{mmole} / \mathrm{l}$, while the chemically modified cyclodextrins concentrations were increased from 5 to $15 \mathrm{mmole} / 1$. Stock solution of drug in methanol \& stock solution of chemically modified CDs using water were prepared in the study. Aliquots from each solution were transferred to a $25 \mathrm{ml}$ volumetric flask and the volume was made up using distilled water so that the required concentrations are obtained. The prepared solutions were stirred for $2 \mathrm{~h}$, filtered through $0.451 \mu$ membrane filter (Ustar LB, USA), and the UV absorption spectra were recorded (UV spectrophotometer-1601 PC, Shimadzu, Japan) in the wavelength range from 200 to $400 \mathrm{~nm}$ vs blank solutions containing the same concentrations of cyclodextrins. The recorded spectra were compared to the spectrum of free nelfinavir.

Phase solubility studies. The effects of CDs on the solubility of nelfinavir were investigated according to the phase solubility technique established by Higuchi and Connors. ${ }^{9}$ An excess amount of drug $(50 \mathrm{mg}$ ) were added to $25 \mathrm{ml}$ portions of distilled water, each containing variable amount of HP- $\beta-C D$ such as 3, 6, 9, 12 and $15 \times 10^{-3}$ moles/liter in $50 \mathrm{ml}$ Stoppard glass bottles.. All the above solutions were shaken for $72 \mathrm{hrs}(100 \mathrm{rpm})$ at $25 \pm 0.5^{\circ} \mathrm{C}$ in a thermostatically controlled shaking water bath (Model 1083, Remi). After equilibrium attainment, aliquots were withdrawn; solutions were filtered and assayed spectrophotometrically (UV1700, Shimadzu, Japan) for nelfinavir content at 254 $\mathrm{nm}$ against blank solutions containing the same concentrations of cyclodextrins. Each experiment was carried out in triplicate. Phase solubility diagrams were obtained by plotting the solubility of nelfinavir, in $\mathrm{mM}$, versus the concentrations of the cyclodextrins used. The complexation efficiency (CE), which reflects the solubilizing power of the CDs towards the drug, was calculated from the 
straight line of the phase solubility diagrams according to the equation. ${ }^{10}$

$$
\mathrm{K}_{(1: 1)}=\frac{\text { Slope }}{\mathrm{S}_{0}(1-\text { slope })}
$$

The slope is obtained from the initial straightline portion of the plot of NFV concentrations against HP- $\beta-C D$ concentration. $S_{0}$ represents the drug solubility in the absence of cyclodextrins, $\mathrm{K}_{1: 1}$ is the apparent stability constant.

Preparation of nelfinavir-CD solid binary systems. Solid inclusion complexes of nelfinavir with HP- $\beta$-CD were prepared using kneading, coprecipitation and freeze-drying techniques in molar ratios of 1:1, 1:2, 1:3 \& 1:5 (drug to $C D$ ). Physical mixtures were also prepared in the same molar ratios for comparison.

Physical mixtures. Physical mixtures of nelfinavir and CDs were prepared by thoroughly mixing the two components in a mortar for $30 \mathrm{~min}$. with constant trituration, passed through sieve no.100 and stored in desiccators over fused calcium chloride.

Kneading method. The calculated amounts of nelfinavir and cyclodextrin were accurately weighed. First cyclodextrin is added to the mortar, small quantity of 50\% ethanol (water: ethanol; 50:50; v/v) is added while triturating to get slurry like consistency. Then slowly drug is incorporated into the slurry and trituration is further continued for one hour. Slurry is then air dried at $25^{\circ} \mathrm{C}$ for $24 \mathrm{hrs}$, pulverized and passed through sieve no.100 and stored in desiccators over fused calcium chloride as a dehydrating agent.

Co-precipitation method. NFV was dissolved in ethanol at room temperature and HP- $\beta-C D$ was dissolved in distilled water. Different molar ratios of NFV and HP- $\beta-C D$ were taken respectively. The mixture was stirred at room temperature respectively for one hour and then slowly evaporated on a boiling water bath. The inclusion complex precipitated as a crystalline powder was pulverized and passed through sieve no.100 and stored in desiccators till free from any traces of the organic solvent.
Freeze-drying method. Freeze-drying monophase solution method was applied. Solutions were prepared separately by mixing appropriate quantities of cyclodextrins and nelfinavir dissolved in distilled water and glacial acetic acid, respectively. The resulting solutions were mixed by stirring. The clear monophase solution was frozen at $-20{ }^{\circ} \mathrm{C}$, and subsequently freeze-dried for $24 \mathrm{~h}$ at $-50{ }^{\circ} \mathrm{C}$ using a freeze-dryer.

Physicochemical characterization of nelfinavir-CD solid binary system. DSC thermograms, X-ray diffractograms and FTIR spectra were recorded for pure nelfinavir, pure cyclodextrins, and their binary systems prepared by different techniques in molar ratio of 1:5 (drug to $\mathrm{CD}$ ).

Differential scanning calorimetry. DSC using a Mettler Toledo SR system analyzed the samples. The apparatus was calibrated with purified indium (99.9\%). The samples (6 mg each) were placed into pierced aluminum sample container. The studies were performed under static air atmosphere in the temperature range of $20^{\circ} \mathrm{C}$ to $450^{\circ} \mathrm{C}$, at a heating rate of $10^{\circ} \mathrm{C} /$ minutes. The peak temperatures were determined after calibration with standard.

Powder X-ray diffractometry. The X-RD patterns of drug, HP- $\beta-C D \&$ complexes were recorded at room temperature by using an automated Philips PW 1729 X-ray generator with filter Ni, Cu radiation over intervals $10-80^{\circ} / 2 \theta$. The samples were irradiated with Ni-filtered $\mathrm{Cu}$ Ka radiation, at $45 \mathrm{kV}$ voltages and $40 \mathrm{~mA}$ current. The operation data were follows: -filter- $\mathrm{Ni}, \mathrm{x}$ ray target- $\mathrm{Cu}$, wavelength$1.542 \mathrm{~A}$ and scanning speed $1 \% \mathrm{~min}$.

Fourier transform infrared (FTIR) spectroscopy. The FTIR spectra were recorded using a JASCOFTIR-5300 spectrophotometer of NFV and their complexes were obtained by $\mathrm{KBr}$ pellet method. Infrared spectroscopy is one of the most powerful analytical techniques that offer the possibility of chemical identification. The various IR spectra of inclusion complexes prepared by physical mixture, kneading method, freeze drying and coprecipation method were obtained and studied for interaction patterns. The smoothing of the spectra and the 
baseline correlation procedures were applied. The FTIR measurements were performed in the scanning range of $4000-400 \mathrm{~cm}^{-1}$ at ambient temperature.

In vitro dissolution studies. The in-vitro dissolution of the complex was compared with those of pure drug and physical mixtures. The dissolution rate studies were carried out in $900 \mathrm{ml}$ of distilled water and phosphate buffer $(\mathrm{pH}=4)$ using USPXXIII dissolution apparatus (Electrolab) with a peddle stirrer, according to United States Pharmacopoeia (USP). The complex equivalent to $250 \mathrm{mg}$ NFV of the prepared systems was spread on the surface of the dissolution medium. The dissolution medium was $900 \mathrm{ml}$ of distilled water \& phosphate buffer $(\mathrm{pH}=4)$. The stirring speed was $100 \mathrm{rpm}$, and the temperature was maintained at $37 \pm 0.5^{\circ} \mathrm{C}$. At selected time intervals for a period of $120 \mathrm{~min}$, aliquots each of 5 $\mathrm{ml}$ were withdrawn from the dissolution medium through a $0.45 \mu \mathrm{m}$ membrane filter and replaced with an equivalent amount of the fresh dissolution medium. Samples were analyzed for drug release by measuring the absorbance at $254 \mathrm{~nm}$ (after suitable dissolution with distilled water or phosphate buffer) using the regression equation of a standard curve developed in the same medium. Each experiment was carried out in triplicate. ${ }^{11}$ Along with this the dissolution profiles were evaluated on the basis of the dissolution efficiency parameter at $60 \mathrm{~min}\left(\mathrm{DE}_{60}, \%\right)$, calculated from the area under the dissolution curves and expressed as a percent of the area of the rectangle described by $100 \%$ dissolution in the same time. ${ }^{12}$

\section{RESULTS AND DISCUSSION}

UV spectroscopic measurements: Increasing the concentration of all CDs from 5 to $15 \mathrm{mmole} / \mathrm{L}$ resulted in an increase in the absorbance of nelfinavir without any shifts of $\lambda$ max. Figures a $\& b$ show the effect of CDs concentrations on the absorption spectra of nelfinavir in aqueous solutions. The observed red/hyperchromic shift might be due to the perturbation of the chromophore electrons of the drug due to the inclusion into the cyclodextrin cavity. ${ }^{13}$ It could be indicative of cyclodextrin guest-host complex formation. ${ }^{13,14}$

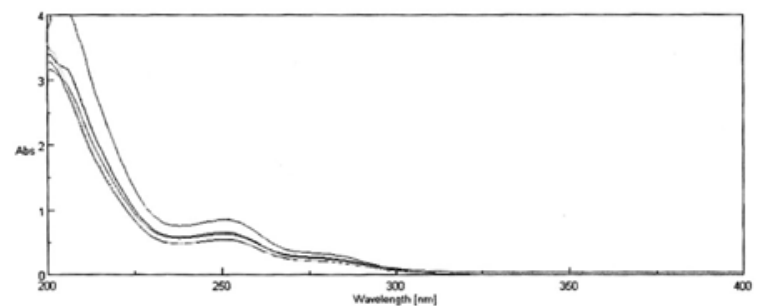

Figure a. Differential ultraviolet absorbance spectra of nelfinavir in presence of $\beta-C D$

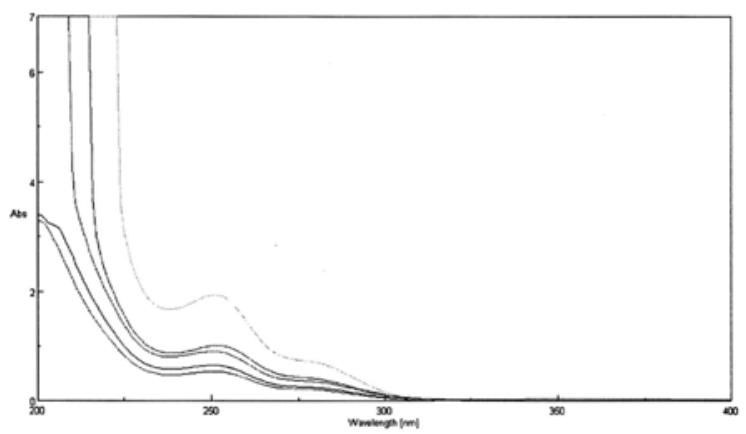

Figure b. Differential ultraviolet absorbance spectra of nelfinavir in presence of HP- $\beta-C D$.

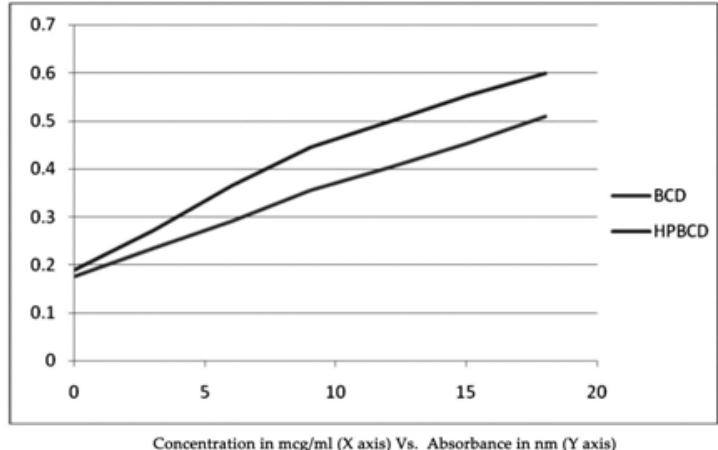

Figure 1. Phase solubility diagrams of nelfinavir with different cyclodextrins in distilled water at $37 \pm 0.5^{\circ} \mathrm{C}$

Phase solubility studies. The phase solubility diagrams of nelfinavir with HP- $\beta-\mathrm{CD}$ in distilled water at $37 \pm 0.5^{\circ} \mathrm{C}$ are shown in Figure 1. The solubility of nelfinavir increased as a function of the CDs concentrations due to the formation of cyclodextrin complexes. ${ }^{9} \quad$ Moreover, other interactions may be involved, such as aggregation of cyclodextrins and their complexes into water soluble aggregates that are capable of solubilizing water insoluble drugs via noninclusion complexation or micelle-like structure. ${ }^{15}$ The coefficient of determination $\left(\mathrm{r}^{2}\right)$ value of the phase solubility diagram with CDs was $<0.990$ (0.983 for HP- $\beta-C D$ ); 
therefore, the diagram was classified as $A_{P}$-type curve. ${ }^{16}$ This positive deviation from linearity could suggest the formation of higher order inclusion complexes with respect to cyclodextrin. ${ }^{17}$

Preparation of nelfinavir-CD solid binary systems. Solid binary systems of nelfinavir using HP- $\beta-C D$ in molar ratios of 1:1, 1:2, 1:3 \&1:5 (drug to $\mathrm{CD}$ ) were prepared using kneading, co precipitation and freeze-drying techniques. Based on the results obtained through the phase solubility studies, which proved the possibility of formation of higher order complexes between nelfinavir and chemically modified cyclodextrins, viz. 1:5 (drug to CD) molar ratios were chosen for the preparation of solid binary systems in addition to the conventional 1:1 molar ratio physical mixtures were also investigated in the same molar ratios for comparison.

Differential scanning calorimetry (DSC). The thermal behavior of cyclodextrin inclusion complex was studied using DSC in order to confirm the formation of solid inclusion complexes. The thermograms of pure NFV, HP- $\beta-C D$ and corresponding cyclodextrin complexes represented in Figure 2. The DSC thermogram of nelfinavir was typical of a crystalline substance, exhibiting a sharp endothermic peak at ad $316.69^{\circ} \mathrm{C}$ corresponding to the melting point of the drug. The drug endothermic melting peak completely disappeared in the DSC thermograms of the freeze-dried systems (FD) prepared using HP- $\beta-C D$. This could indicate amorphous solid dispersion or molecular encapsulation of the drug into the cyclodextrin cavity. ${ }^{18}$

However, all other investigated systems have recorded the drug melting endotherm, but with marked broadening and reduction in intensity. In case of HP- $\beta-C D$ the marked broadening which was observed might indicates the masking of the drug melting endotherm due to the overlapping of the vicinity of the two effects. ${ }^{19}$ Also a considerable reduction in the intensity of the melting peak in the aforementioned systems could be attributed to the low drug to CD molar ratio (1:3) (the drug content of the prepared systems did not exceed $10 \%$ by weight of the system). Though, the DSC could not be considered as a discriminative analysis tool between these systems, the existence of the drug melting peak in these systems could suggest that no true inclusion is formed in either of these systems.
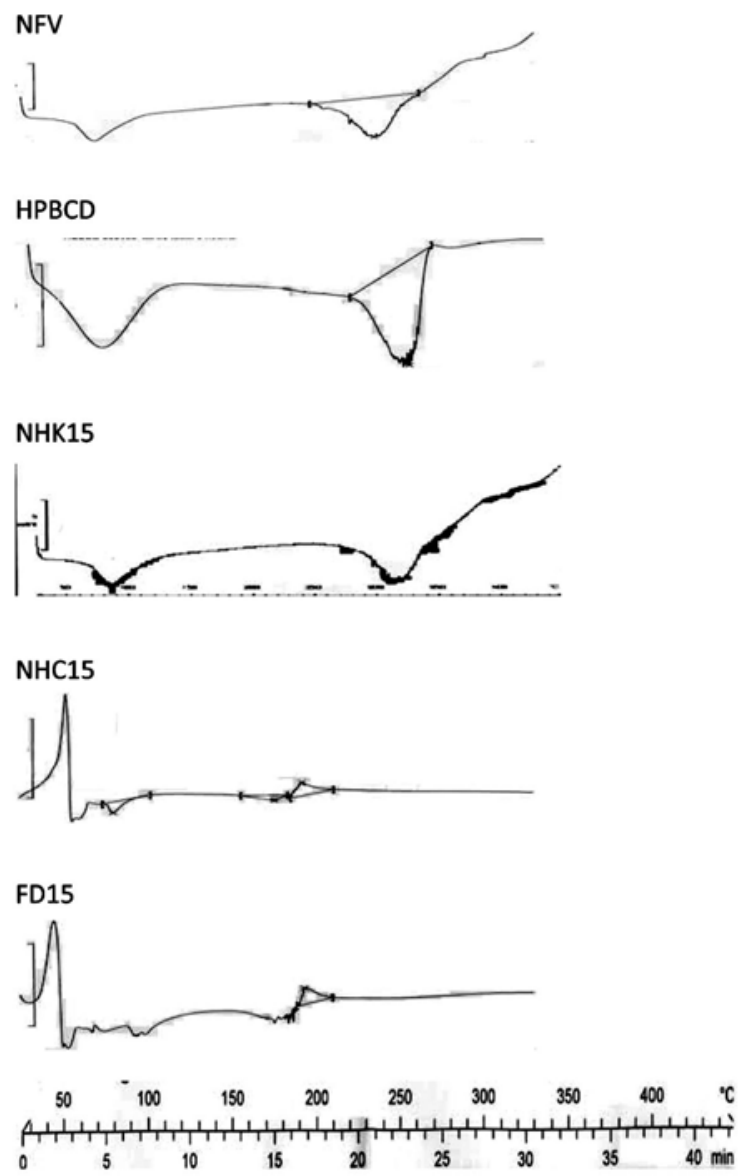

Figure 2. DSC thermograms of pure drug, polymer \& formulation (NHK1, NHP1, NHC1, pure drug \& HPBCD).

X-ray diffractometry (XRD). XRD was performed for the pure drug, pure cyclodextrins and their binary systems prepared by different techniques at a molar ratio of 1:5 (drug to $\mathrm{CD}$ ) (Figures not shown). The diffraction pattern of nelfinavir powder revealed several sharp high intensity peaks at diffraction angles $(2 \theta)$ suggesting that the drug existed as crystalline material. Similar observations have been reported by other authors. ${ }^{20-21}$ The diffraction patterns of the investigated physical mixtures correspond to the superposition of those of the pure components. During mixing and dilution of 
the pure crystalline components lower intensities of the diffraction peaks were observed due to particle size reduction. Overlapping of some nelfinavir diffraction peaks with those of pure $\mathrm{CD}$ was evident. The diffractograms of the KN systems showed almost similar diffraction behavior to the coprecipation method. But the crystallinity of nelfinavir was higher in the kneaded nelfinavir -HP- $\beta$-CD than the corresponding coprecipation binary system prepared. The presence of nelfinavir peaks in the diffractogram of nelfinavir: HP- $\beta-C D$, freeze-dried system could suggest indicating the entirely amorphous nature, although reduction in number and intensities were observed. Therefore, since the amorphization of the drug can be a sequence of the lyophilization process, it is possible that the X-ray data cannot discriminate whether the drug-CD lyophilized systems obtained are true inclusion complexes or homogenous dispersed mixtures of the amorphous components. ${ }^{22}$ As per Williams et al., lack of crystallinity is an added evidence for the formation of inclusion complex. ${ }^{23}$

Fourier-transform infrared spectroscopy (FTIR). The FTIR spectra of pure components and their binary systems prepared by different techniques at molar ratio of 1:5 (drug to $\mathrm{CD}$ ) are shown in Figure 3. The FTIR spectrum of nelfinavir showed characteristic absorption band for $-\mathrm{OH},-\mathrm{NH}$ and $\mathrm{C}=\mathrm{O}$ stretching at $3613 \mathrm{~cm}^{-1}, 3396.95 \mathrm{~cm}^{-1} 1647.36 \mathrm{~cm}^{-1}$ and $3522.34 \mathrm{~cm}^{-1}, 3354.51 \mathrm{~cm}^{-1}, 1649.29 \mathrm{~cm}^{-1}$ respectively when compared with the characteristic peak values of pure drug. This shift in the values of peaks indicates the interaction between drug and polymer. Two intense absorption bands attributed to the carbonyl stretching vibration were found at 1647.36 and $1649.29 \mathrm{~cm}^{-1}$. The FTIR spectra of the pure chemically modified CDs illustrated intense broad absorption bands at $3600-3300 \mathrm{~cm}^{-1}$ corresponding to the free $-\mathrm{OH}$ stretching vibration. Moreover, the characteristic $-\mathrm{NH}$ stretching band of nelfinavir was masked in all the prepared systems by the broad intense band corresponding to the free $-\mathrm{OH}$ vibration of CDs. This effect was expected due to the low drug to $C D$ molar ratio (1:5). Along with this, there was an overlap between the carbonyl stretching

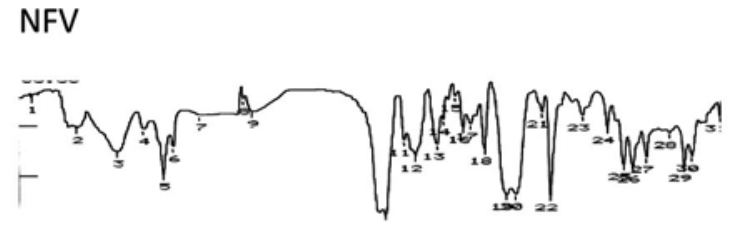

$H P \beta-C D$
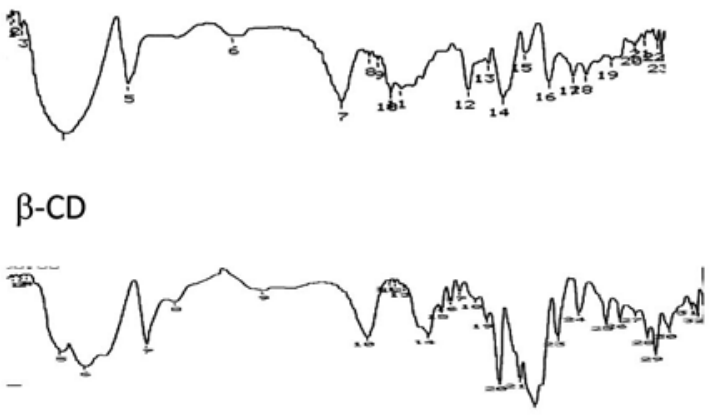

NHP15

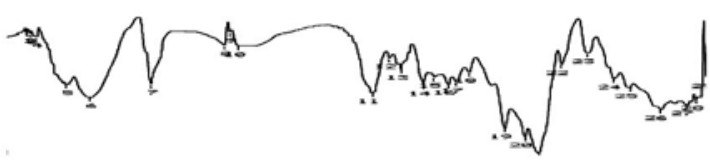

NHK15

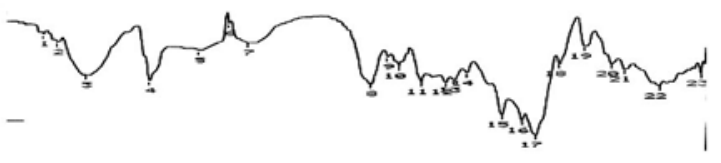

NHC15

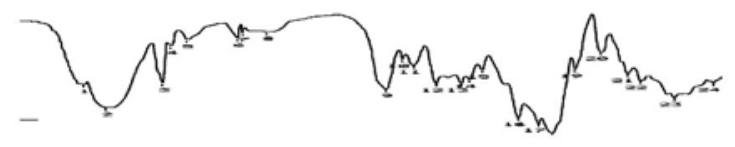

FD15

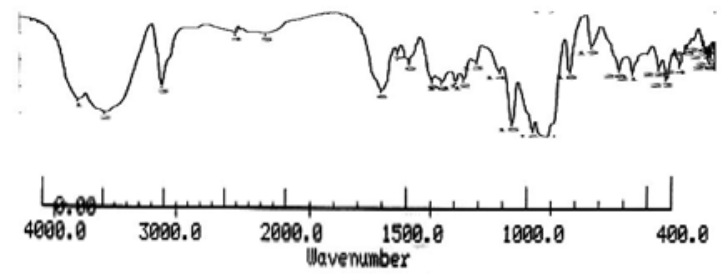

Figure 3. FTIR spectrum of pure drug, polymer \& various formulations.

of the drug at $1649 \mathrm{~cm}^{-1}$ and the band corresponding to the hydrated bonds within CD molecules at 1650$1640 \mathrm{~cm}^{-1}$. Therefore the main characteristic band 
used to assess the drug-CD interactions was the carbonyl stretching of nelfinavir at $1647.36 \mathrm{~cm}^{-1}$

With respect to the FTIR spectra of the pure components, and in particular the characteristic carbonyl stretching band of nelfinavir, the FTIR spectra of all the PMs and the KN products did not show any significant changes. Compared to this the FTIR spectra of the FD products exhibited a shift of the characteristic nelfinavir carbonyl stretching band to higher frequencies up to $1812 \mathrm{~cm}^{-}{ }^{1}$, with decrease in intensity. Such kind of shifts has been reported for other drugs, and was explained by the dissociation of the intermolecular hydrogen bonds associated with crystalline drug molecules. ${ }^{418}$ This indicates possibility of nelfinavir dispersion due to interaction with CDs, which could result in its inclusion in the hydrophobic cavity of the carrier. The overall decrease in the intensity and broadening of the drug carbonyl stretching band observable in these systems might be due to its restriction within the cyclodextrin cavity. ${ }^{24}$

In vitro dissolution studies. The dissolution profiles of nelfinavir from its solid drug-CD binary systems in distilled water \& phosphate buffer are demonstrated in Figures $4 \& 5$. The percent amount dissolved was calculated according to predetermined drug content for each product. The initial dissolution rate during the first $5 \mathrm{~min}$ (IDR) and the extent of dissolution after 60 min $\left(\mathrm{DP}_{60}\right)$ of all the systems are compiled in Tables 1 and 2.

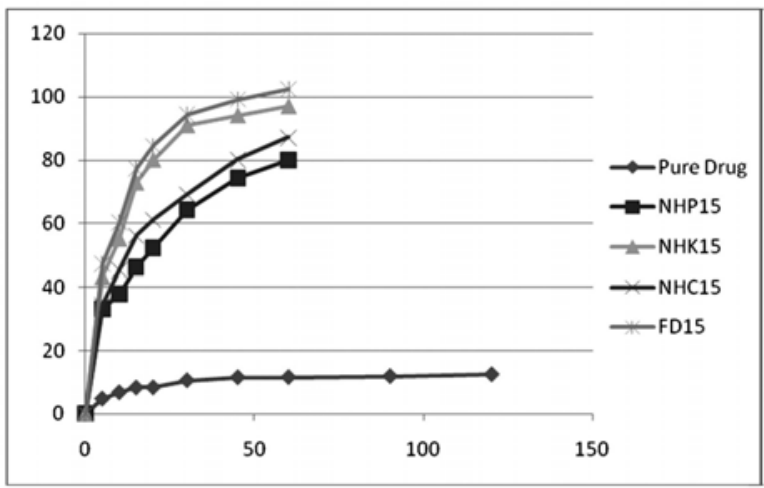

Time (min) [X axis] Vs \% Drug Dissolved \pm SD [Y axis]

Figure 4. Dissolution profiles of nelfinavir from nelfinavir: HP- $\beta$ $\mathrm{CD}$ binary systems in distilled water at $37 \pm 0.5^{\circ} \mathrm{C}$ (mean $\pm \mathrm{SD}, \mathrm{n}$ $=3$ ).

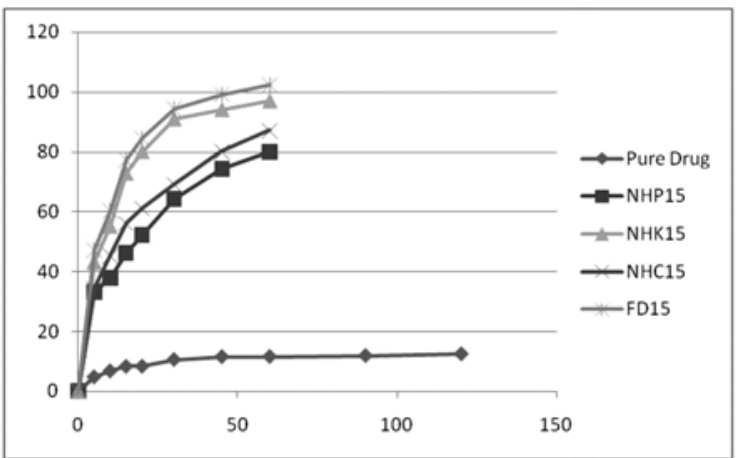

Time (min) [X axis] Vs \% Drug Dissolved $\pm \mathrm{SD}[\mathrm{Y}$ axis]

Figure 5. Dissolution profiles of nelfinavir from nelfinavir: HP- $\beta$ $\mathrm{CD}$ binary systems in phosphate buffer $(\mathrm{pH}=4)$ at $37 \pm 0.5{ }^{\circ} \mathrm{C}$ (mean $\pm \mathrm{SD}, \mathrm{n}=3$ ).

It was found that for pure nelfinavir no dissolution was achieved even after $120 \mathrm{~min}$, under the specified dissolution conditions. Drug was found to float on the surface due to the hydrophobic property of the drug which prevented its contact with the dissolution medium.

Since CDs dissolve in a short time it was found responsible for the dissolution enhancement of nelfinavir when physically mixed with $\mathrm{CDs}$ due to local solubilization action of the carrier operating in the microenvironment of the drug, or the hydrodynamic layer surrounding drug particles in the early stages of the dissolution process owing to an insitu inclusion process causing a rapid increase in the amount of dissolved drug.

It was found that the increase in the rate of drug dissolution was to a lesser extent from physical mixtures. The reason for this could be attributed to the surfactant-like properties of cyclodextrins, which reduce the interfacial tension between the water insoluble drug particles and the dissolution medium, thus improving the wettability and dissolution of the drug. ${ }^{25}$ Compared to the PMs kneading (KN) products showed somewhat better dissolution behavior of the physical mixtures (PMs) with limited enhancement in nelfinavir dissolution. This slight increase in the dissolution of the $\mathrm{KN}$ method is due to employing a semisolid medium during the systems preparation, where the interactions between the drug and the cyclodextrin might be limited ${ }^{2,25}$ The better drug dissolution compared to the PMs is probably due to the increase in the drug-carrier contact surface 
as a consequence of the more drastic mechanical treatment ${ }^{2}$. The co-precipitation method has shown almost similar result as that of the PMs.

Freeze dried (FD) systems showed marked increase in nelfinavir dissolution compared with the other methods, especially for the water soluble HP$\beta$-CD derivatives, showing a burst effect of more than $70 \%$ during the first $5 \mathrm{~min}$. This could be mainly due to the formation of soluble inclusion complexes of the drug with chemically modified CDs and the high energetic amorphous state or reduction of the crystallinity following complexation as reported previously. ${ }^{26}$ This marked increase in the dissolution rate might be due to the formation of solid solution of the drug in the FD products as a result of the complete inclusion of the drug into the CD cavities.
The molecular size of the particle could be the reason behind fast dissolution, which causes the carrier, brought the drug into the dissolution medium. The use of glacial acetic acid in freeze-drying technique was found to improve nelfinavir complexation with CDs. Johnson et al. in his work used glacial acetic acid as a solvent to improve cyclodextrin complexation with an anti-hepatitis drug. ${ }^{27}$ Moreover, Loftsson et al. used glacial acetic acid to enhance the complexation efficiency of cyclodextrins with water insoluble basic drugs through drug ionization in aqueous medium. ${ }^{28}$ Owing to volatility and miscibility with water in all proportions, acetic acid is considered as a good candidate for freeze-drying medium. $^{28}$

Table 1. Dissolution parameters of different nelfinavir-CD binary systems

\begin{tabular}{|c|c|c|c|c|c|c|c|c|c|}
\hline \multirow{2}{*}{$\begin{array}{l}\text { Nelfinavir-CD } \\
\text { system }\end{array}$} & \multirow{2}{*}{$\begin{array}{l}\text { Molar } \\
\text { ratio }\end{array}$} & \multicolumn{4}{|c|}{ Initial dissolution rate (IDR \% dissolved/min) } & \multicolumn{4}{|c|}{ Extent of dissolution after $60 \mathrm{~min}\left(\mathrm{DP}_{60} \%\right)$} \\
\hline & & PM & $\mathrm{KN}$ & $\mathrm{CP}$ & FD & PM & $\mathrm{KN}$ & $\mathrm{CP}$ & FD \\
\hline \multirow{4}{*}{$\begin{array}{l}\text { Nelfinavir-HP- } \\
\beta-\mathrm{CD}\end{array}$} & $1: 1$ & $1.09 \pm 0.04$ & $2.32 \pm 0.05$ & $1.23 \pm 0.14$ & $3.77 \pm 0.15$ & $12.77 \pm 1.02$ & $22.43 \pm 1.32$ & $13.32 \pm 1.01$ & $29.89 \pm 0.02$ \\
\hline & $1: 2$ & $1.88 \pm 0.16$ & $3.32 \pm 0.11$ & $1.93 \pm 0.23$ & $6.67 \pm 0.04$ & $14.44 \pm 0.54$ & $24.59 \pm 0.34$ & $14.84 \pm 0.42$ & $38.32 \pm 0.04$ \\
\hline & $1: 3$ & $2.78 \pm 0.19$ & $4.21 \pm 0.23$ & $2.98 \pm 0.06$ & $9.89 \pm 0.07$ & $17.43 \pm 0.87$ & $27.89 \pm 0.52$ & $17.99 \pm 0.43$ & $49.23 \pm 0.04$ \\
\hline & $1: 5$ & $3.32 \pm 0.22$ & $5.54 \pm 0.47$ & $3.69 \pm 0.33$ & $14.43 \pm 0.12$ & $18.99 \pm 0.35$ & $29.34 \pm 0.43$ & $19.43 \pm 1.42$ & $69.23 \pm 1.78$ \\
\hline
\end{tabular}

HP- $\beta-\mathrm{CD}$, hydroxypropyl beta cyclodextrin; PM, physical mixture; KN, kneaded method; CP, co-precipitation method; FD, freeze-dried method.

Table 2. DE60 of different nelfinavir-CD binary systems

\begin{tabular}{lccccc}
\hline Nelfinavir-CD system & Molar ratio & \multicolumn{4}{c}{ Dissolution efficiency (DE60 \%) } \\
\cline { 3 - 5 } & & PM & KN & CP & FD \\
\hline Nelfinavir-HP- $\beta$-CD & $1: 1$ & $10.44 \pm 0.34$ & $18.38 \pm 1.07$ & $11.23 \pm 0.02$ & $27.32 \pm 1.57$ \\
& $1: 2$ & $13.89 \pm 0.65$ & $22.64 \pm 0.54$ & $13.22 \pm 0.09$ & $34.59 \pm 0.03$ \\
& $1: 3$ & $16.99 \pm 0.43$ & $27.86 \pm 0.63$ & $16.12 \pm 1.03$ & $48.24 \pm 1.43$ \\
& $1: 5$ & $18.99 \pm 0.52$ & $32.64 \pm 1.04$ & $19.54 \pm 1.14$ & $74.22 \pm 0.06$ \\
\hline
\end{tabular}

(Values are mean $\pm \mathrm{SD}, \mathrm{n}=3$ )

The increment in drug dissolution from nelfinavir: HP- $\beta-C D$ systems with 1:5 proportion was higher than the corresponding ones with nelfinavir: HP- $\beta-C D$ with $1: 1$, probably due to stronger interaction between nelfinavir and HP- $\beta$-CD and/or better inclusion of the drug molecules into the CD hydrophobic cavity that expanded by the two methyl groups. ${ }^{29}$ Besides the preparation method and the drug to $\mathrm{CD}$ molar ratio, the effect of the CD type was also evident on the dissolution of nelfinavir, where the solid binary systems prepared using different concentrations of HP- $\beta-C D$ exhibited superior enhancement in nelfinavir dissolution compared with that prepared using the parent CDs, especially on using the freeze-dried technique. The reason could be explained on the basis of better wetting ability, greater water solubility, and higher complexing power of CDs derivatives towards the drug in the solid state. 


\section{CONCLUSION}

Solid binary systems of nelfinavir with chemically modified cyclodextrin like HP- $\beta$-CD were prepared using physical mixtures, kneading, coprecipitation method and freeze-drying techniques in 1:1, 1:2, 1:3 and 1:5 (drug: CD) molar ratios. From the above results, it is possible to conclude that HP- $\beta$ -CD will able to form true inclusion complexes with nelfinavir at a molar ratio of 1:5 using the freezedrying technique. The dissolution of nelfinavir was markedly enhanced in both systems, showing an initial burst effect of more than $75 \%$ in the first 5 min. Hence, the FD system of nelfinavir with HP- $\beta$ CD prepared at a molar ratio of 1:5 could be chosen for the formulation of nelfinavir tablets due to the well-documented safety profile of HP- $\beta$-CD.

\section{ACKNOWLEDGEMENTS}

This work was supported by the principal, HKES'S College of Pharmacy, Gulbarga, Karnataka. Authors are grateful to the Dr. M.G. Purohit for giving his valuable contribution during the work in various areas. We also thank Mr. Gogai for giving his technical support during the preparation of the manuscript.

\section{REFERENCES}

1. Agouron Pharmaceuticals. 2008. Viracept (nelfinavir mesylate) tablets and oral powder prescribing information. La Jolla, CA; Sep.

2. Fernandes, C., Teresa V. and Veiga, F. 2002. Physicochemical characterization and in vitro dissolution behavior of nicardipine-cyclodextrins inclusion compounds. Eur. J. Pharm. Sci. 15, 79-88.

3. Nagarsenker, M. and Joshi, M. 2005. Celecoxib-cyclodextrin systems: characterization and evaluation of in vitro and in vivo advantage. Drug Dev. Ind. Pharm. 31, 169-178.

4. Rajendrakumar, K., Madhusudan, S., and Pralhad, T. 2005. Cyclodextrin complexes of valdecoxib: properties and antiinflammatory activity in rat. Eur. J. Pharm. Biopharm. 60, 39-46.

5. Jun, S., Kim, M., Kim, J., Park, H., Lee, S., Woo, J. and Hwang, S. 2007. Preparation and characterization of simvastatin/hydroxypropyl-b-cyclodextrin inclusion complex using supercritical antisolvent (SAS) process. Eur. J. Pharm. Biopharm. 66, 413-421.
6. Rajewski, R. and Stella, V. 1996. Pharmaceutical applications of cyclodextrins. 2: In vivo drug delivery. $J$. Pharm. Sci. 85, 1142-1169.

7. Szejtli J. 1988.Cyclodextrin Technology. Dordrecht, The Netherlands: Kluwer Academic Publishers; 81-83.

8. Connors, K. and Mollica, J. 1966.Theoretical analysis of comparative studies of complex formation. J. Pharm. Sci. 55, 772-780.

9. Higuchi, T. and Connors, K. 1965.Phase solubility techniques, in: C.N. Reilly (Ed.), Advances in Analytical and Chemistry Instrumentation. Wiley Interscience, New York, 4, 117-212.

10. Loftsson, T., Masson, M. and Sigurjonsdottir, J. 1999. Methods to enhance the complexation efficiency of cyclodextrin. STP Pharma Sci. 9, 237-242.

11. Habib, M., Phan, M. and Owusu-Ababio, G. 1998. Dissolution profiles of flurbiprofen in phospholipids solid dispersions. Drug Dev. Ind. Pharm. 24, 1077-1082.

12. Khan, K. and Rhodes, C. 1972. Effect of compaction pressure on the dissolution efficiency of direct compression systems, Pharm. Acta Helv. 47, 594-607.

13. Ventura, C., Giannone, I., Musumeci, T., Pignatello, R., Ragni, L., Landolfi, C., Milanese, C., Paolino, D. and Puglisi, G. 2006. Physico-chemical characterization of disoxarildimethyl- $\beta$-cyclodextrin inclusion complex and in vitro permeation studies. Eur. J. Med. Chem. 41, 233-240.

14. Crupi, V., Ficarra, R., Guardo, M., Majolino, D., Stancanelli, R. and Venuti, V. 2007. UV-vis and FTIR-ATR spectroscopic techniques to study the inclusion complexes of genistein with $\beta$-cyclodextrins. J. Pharm. Biomed. Anal. 4, 110-117.

15. Loftsson, T., Masson, M. and Brewster, M. 2004. Self association of cyclodextrin and cyclodextrin complexes. $J$. Pharm. Sci. 93, 1091-1099.

16. Arima, H., Yunomae, K., Miyake, K., Irie, T., Hirayama, F. and Uekama, K. 2001. Comparative studies of the enhancing effects of cyclodextrins on the solubility and oral bioavailability of tacrolimus in rats. J. Pharm. Sci. 90, 690701.

17. Loftsson, T., Magnusdottir, A., Masson, M. and Sigurjonsdottir, J. 2002. Self association and cyclodextrin solubilization of drugs. J. Pharm. Sci. 91, 2307-2316.

18. Mura, P., Adragna, E., Rabasco, A., Moyano, J., PerezMartı'nez. J., Arias, M. and Gines, 1999. J. Effects of the host cavity size and the preparation method on the physicochemical properties of ibuproxam-cyclodextrin systems. Drug Dev. Ind. Pharm. 25, 279-287.

19. Liu, J., Qui, L. and Gao, J. 2006. Jin Y. Preparation, characterization and in vivo evaluation of formulation of baicalein with hydroxypropyl-b-cyclodextrin. Intl. J. Pharm. 312, 137-143. 
20. Badr-Eldin, S., Elkheshen, S. and Ghorab, M. 2008. Inclusion complexes of tadalafil with natural and chemically modified b-cyclodextrins. I: Preparation and in-vitro evaluation. Eur. J. Pharm. Biopharm. 70, 819-827.

21. Manosroi, J., Apriyani, M., Foe, K. and Manosroi, A. 2005. Enhancement of the release of azaleic acid through the synthetic membranes by inclusion complex formation with hydroxypropyl-b-cyclodextrin. Intl. J. Pharm. 293, 235-240.

22. Redenti, E., Peveri, T., Zanol, M., Ventura, P., Gnappi, G. and Montenero, A. 1996. A study on the differentiation between amorphous piroxicam:b-cyclodextrin complex and a mixture of the two amorphous components. Intl. J. Pharm. 129, 289-294.

23. Williams, R., Mahaguna, V. and Sriwongjanya, M. 1998. Characterization of a inclusion complex of cholesterol and hydroxypropyl-b-cyclodextrin. Eur. J. Pharm. Biopharm. 46, 355-360.

24. Ficarra, R., Ficarra, P., Di Bella, M., Raneri, D., Tommasini, S., Calabro, M., Villari, A. and Coppolino, S. 2000. Study on the inclusion complex of atenolol with bcyclodextrin. $J$. Pharm. Biomed. Anal. 23, 231-236.
25. Moyano, J., Gines, J., Arias, M. and Rabasco, A. 1995. Study of the dissolution characteristics of oxazepam via complexation with b-cyclodextrin. Intl. J. Pharm. 114, 95-102.

26. Dollo, G., Corre, P., Chollet, M,, Chevanne, F., Bertault, M., Burgot, J. and Verge, R. 1999. Improvement in solubility and dissolution rate of 1,2-dithiole-3-thiones upon complexation with b-cyclodextrin and its hydroxypropyl and sulfobutyl ether-7 derivatives. J. Pharm. Sci. 88, 889-895.

27. Johnson, J., He, Y., Jain, A. and Yalkowsky, S. 2006. Improving cyclodextrin complexation of a new antihepatitis drug with glacial acetic acid. AAPS Pharm. Sci. Tech. 7, E1E6. article 18.

28. Loftsson, T., Sigurdsson, H., Masson, M. and Schipper, N. 2004. Preparation of solid drug/cyclodextrin complexes of acidic and basic drugs. Pharmazie 59, 25-29.

29. Ahmed, M. 2001. Comparison of impact of the different hydrophilic carriers on the properties of piperazinecontaining drug. Eur. J. Pharm. Biopharm. 51, 221-226. 